\title{
P.6 ESTIMATIONS OF MODEL PARAMETERS FOR GRAVITY WAVE SPECTRA OBSERVED BY MST RADAR
}

\author{
A. O. Scheffler, C. H. Liu, and S. J. Franke \\ Department of Electrical and Computer Engineering \\ University of Illinois, Urbana, IL 61801
}

\begin{abstract}
The general theory of MST radar observations of gravity wave spectra is developed in this paper. This effort extends the previous results to include anisotropy and Doppler effects for the spectra, as well as the consequences for the multibeam configuration. The relationships between the observed one- or two-dimensional spectra for the line-of-sight velocity in the gravity wave spectra are derived. Expressions for cross spectra, as well as covariances between velocities observed on different beams, are computed. Using these results, studies are carried out to show how model parameters for gravity wave spectra can be estimated from the observed quantities. Model parameters include the variance, power-law indices, anisotropy parameters, Doppler parameters, mean scale sizes, etc. Cases with different numbers of beams will be investigated.
\end{abstract}

\section{INTRODUCTION}

Recently, MST radars have been used by many investigators to study the spectra of velocity fluctuations in the middle atmosphere [Balsley and Carter, 1982; Meek et al., 1985; Scheffler and Liu, 1985; Scheffler and Liu, 1986; Smith et al., 1985; Franke et al, 1988, VanZandt, 1985]. Attempts have been made by these authors to interpret the observed spectra in terms of internal gravity waves. Based on the Garrett-Munk model spectrum for ocean waves, VanZandt [1985] proposed a similar model spectrum for atmospheric waves. Features of the model spectrum have been compared to observed spectra [Smith et al., 1985; Franke et al., 1988].

In this paper, we generalize the model spectrum for the atmosphere to include different types of asymmetry in the model. We then derive relations between radar observed quantities and the model parameters. Based on these relations, we propose several observational configurations and data processing schemes in order to determine, experimentally, some of the parameters in the model spectrum.

\section{MODEL GRAVITY WAVE SPECTRUM}

For a general GM type spectrum for gravity waves the following form is assumed:

$$
E\left(\phi, k_{z} \cdot \omega\right)=E_{0} A_{z}\left(k_{z}, \omega\right) B(\omega) F(\phi, \omega)
$$

$E_{0}$ is the energy density constant; $k_{z}{ }^{*}$ is the vertical wave number bandwidth; $p$ and $t$ are the frequency and wave number power law indices, respectively. The corresponding three algebraic model functions are defined as

$$
\begin{aligned}
& A_{z}\left(k_{z}, \omega\right)=\left(1+\frac{\omega}{|\omega|} \frac{k_{z}}{\mid k_{z}} S S_{v}\right) \frac{(t-1) k_{z}^{* t-1}}{\left(k_{z}^{*}+\left|k_{z}\right|\right)^{t}} \\
& B(\omega)=\frac{p-1}{\omega_{i}^{1-p}-\omega_{b}^{1-p}}|\omega|-p \text { for } \omega_{i} \leq|\omega| \leq \omega_{b} \text {, and } B(\omega)=0 \text { elsewhere }
\end{aligned}
$$




$$
F(\phi, \omega)=\frac{1+S_{h}}{2} \frac{a}{1-\frac{\omega}{|\omega|}\left(1-a^{2}\right)^{1 / 2} \cos \left(\phi-\phi_{a}\right)}+\frac{1-S_{h}}{2} \frac{a}{1-\frac{\omega}{|\omega|}\left(1-a^{2}\right)^{1 / 2} \cos \left(\phi-\phi_{a}\right)}
$$

where $a=\left(1-e^{2}\right)^{1 / 2}$.

The $A_{2}$ function is based upon the familiar GM 75 vertical wave number function introduced in the symmetric model with bandwidth $\mathrm{k}_{\mathbf{z}}$. However, the vertical symmetry faction $S_{v}$, has been added to model the upward/downward propagation asymmetry of gravity waves for two different cases. The spectral shape is the same for the $\pm \mathbf{k}_{\mathbf{z}}$ (upward/downward) branches, but the levels of these two branches are altered so that they are proportional to $1 \pm S_{\mathrm{v}}$. A physically realistic spectrum requires that $\mid S_{v} I \leq 1$. The extreme example of $S_{v}= \pm 1$ therefore corresponds to solely upward/downward propagation. Any case of $S_{v} \neq 0$ adds one degree of freedom to the model to represent vertical asymmetry.

The B function retains the same symmetric power law form as previously assumed. In comparison to Garrett and Munk, however, a double-sided form is emphasized throughout this paper to be consistent with even symmetry of the four-dimensional wave energy spectrum necessary to represent a real velocity field in the space-time domain. This physical requirement is also the reason for introducing the sign of $\omega$ dependence to the $A$ and $F$ functions in order to model the vertical and azimuthal asymmetries. Complete separability is therefore not possible in the asymmetric spectrum, but $\phi-\mathrm{k}_{\mathrm{z}}$ separation is still assumed. If so desired, however, the model presented here is simple enough to be represented as the sum of four completely separable products when the \pm branches of $\phi, k_{z}$, and $\omega$ are treated individually.

The $\mathrm{F}$ function has been introduced to model the azimuthal dependence of the gravity wave spectrum. This function is essentially the sum of two ellipses in the azimuthal plane whose pairs of foci lie on opposite sides of the origin but along the same axis inclined by the angle $\phi_{\mathrm{a}}$ from the $x$-axis. The eccentricity of both ellipses is $e$, which is determined by the azimuthal shape factor $a$. The azimuthal symmetry factor $S_{h}$ controls the relative sizes of the two ellipses in varying degrees, from waves propagating with bilateral symmetry, $S_{h}=0$, to those propagating only toward one axial direction, $S_{h}=1$. The minimum value for the shape faction is $a=0$, which represents propagation only in the vertical plane inclined by $\phi_{\mathrm{a}}$ from the $x$-axis. Nonzero shape factors lead to general three-dimensional spectra. The case for a maximum value of $a=1$ corresponds to azimuthal isotropy for which $F(\phi, \omega)=1$.

The following normalizations apply to the general model for the functions A, B, and F:

$$
\frac{1}{2} \int_{-\infty}^{\infty} A_{z}\left(k_{z}, \omega\right) d k_{z}=\frac{1}{2} \int_{-\infty}^{\infty} B(\omega) d \omega=\int_{\omega_{i}}^{\omega_{b}} B(\omega) d \omega=\frac{1}{2 \pi} \int_{2 \pi}^{0} F(\phi, \omega) d \phi=1
$$

Finally, the spectrum becomes the symmetric Boussinesq model spectrum for the special case of $S_{v}=0$ and $a=1$.

\section{GRAVITY WAVE SPECTRA AND VARIANCES OBSERVED BY RADAR}

We shall proceed to derive the relation which involve the observed frequency spectrum and velocity variance from a given radar beam and the wave spectrum $E(\vec{k}, \omega)$. For a positioned radar beam, $\vec{r}=r \vec{B}$, with range $r$ and beam direction

$$
\hat{\mathrm{B}}\left(\theta_{\mathrm{B}}, \phi_{\mathrm{B}}\right)=\cos \phi_{\mathrm{B}} \sin \theta_{\mathrm{B}} \hat{\chi}+\sin \phi_{\mathrm{B}} \sin \theta_{\mathrm{B}} \hat{\mathrm{y}}+\cos \theta_{\mathrm{B}} \hat{z}
$$

By closely following the procedure in Scheffler and Liu [1985], the observed velocity fluctuation along the beam direction is found to be 


$$
\overrightarrow{\mathrm{V}}_{\mathrm{ob}}(\mathrm{r}, \mathrm{t})=\overrightarrow{\mathrm{V}}(\mathbf{r B}, \mathrm{t}) \cdot \hat{\mathrm{B}}
$$

where $\vec{V}$ is the velocity fluctuation associated with the waves, including the three components $U$, $\mathrm{V}$ and $\mathrm{W}$.

From (4) and(5), we can derive the frequency spectrum for the observed velocity fluctuation:

$$
\begin{aligned}
\mathrm{E}_{\mathrm{ob}}(\omega) & =\mathrm{EV}_{\mathrm{V}}(\omega) \cos ^{2} \phi_{\mathrm{B}} \sin ^{2} \theta_{\mathrm{B}}+\mathrm{E}_{\mathrm{V}}(\omega) \sin ^{2} \phi_{\mathrm{B}} \sin ^{2} \theta_{\mathrm{B}}+\mathrm{E}_{\mathrm{W}}(\omega) \cos ^{2} \theta_{\mathrm{B}} \\
& +\operatorname{ReEUV}(\omega) \sin 2 \phi_{\mathrm{B}} \sin ^{2} \theta_{\mathrm{B}}+\operatorname{ReE}_{\mathrm{UW}}(\omega) \cos \phi_{\mathrm{B}} \sin 2 \theta_{\mathrm{B}} \\
& \mathrm{Y}+\operatorname{ReEVW}_{\mathrm{W}}(\omega) \sin \phi_{\mathrm{B}} \sin 2 \theta_{\mathrm{B}}
\end{aligned}
$$

where the rectilinear spectral components $E_{U}(\omega), E_{V}(\omega)$. $E_{W}(\omega), E_{U V}(\omega)$, and $E_{v w}(\omega)$ are the autospectra and cross spectra of the velocity components, respectively. They can be related to the general model spectrum for the wave, eq (1), through manipulations following the procedure outlined in Scheffler and Liu /3/. The effects of Doppler shift due to background wind can also be included [Scheffler and Liu, 1986].

The general expression for the velocity covariance $\overline{\mathrm{V}_{\mathrm{ob}}{ }^{2}}$ at a given radar beam can be obtained by integration of the frequency autospectrum

$$
\left\langle\mathrm{V}_{\mathrm{ob}}{ }^{2}\right\rangle=\int_{0}^{\infty} \mathrm{E}_{\mathrm{ob}}(\omega) \mathrm{d} \omega
$$

which yields

$$
\begin{aligned}
<V_{o b^{2}}> & =<U^{2}>\cos ^{2} \phi_{B} \sin ^{2} \theta_{B}+<V^{2}>\sin ^{2} \phi_{B} \sin ^{2} \theta_{B}+<W^{2}>\cos ^{2} \theta_{B} \\
& +<U V>\sin 2 \phi_{B} \sin ^{2} \theta_{B}+<U W>\cos \phi_{B} \sin 2 \theta_{B}+<V W>\sin \phi_{B} \sin 2 \theta_{B}
\end{aligned}
$$

where the rectilinear variance components are

$$
\begin{aligned}
& \left\langle U^{2}\right\rangle=E_{o}\left[1-\frac{2}{p+1} \frac{a+(1-a) \sin ^{2} \phi_{a}}{1+a}\right] \\
& \left\langle V^{2}\right\rangle=E_{o}\left[1-\frac{2}{p+1} \frac{a+(1-a) \cos ^{2} \phi_{a}}{1+a}\right] \\
& \left\langle W^{2}\right\rangle=E_{0} \frac{p-1}{3-p}\left(\frac{\omega_{i}}{w_{b}}\right)^{p-1} \\
& \langle U V\rangle=E_{0} \frac{1}{p+1} \frac{1-a}{1+a} \sin 2 \phi_{a} \\
& \langle U W\rangle=-E_{o} S V^{\prime} S_{h^{\prime}}\left(\frac{1-a}{1+a}\right)^{1 / 2} \frac{\bar{\omega}}{\omega_{b}} \cos \phi_{a} \\
& <V W>=E_{0} S_{V^{\prime}} S_{h^{\prime}}\left(\frac{1-a}{1+a}\right)^{1 / 2} \frac{\bar{\omega}}{\omega_{b}} \operatorname{SIN} \phi_{a}
\end{aligned}
$$


where

$$
\bar{\omega}=\int_{\omega_{i}}^{\omega_{b}} \omega B(\omega) d \omega
$$

\section{PARAMETER ESTIMATION FOR GENERAL MODEL GRAVITY WAVE SPECTRUM}

We will now make use of the variance formulae obtained in the preceding section to outline a parameter estimation procedure for the general model spectrum. Let us assume that we have simultaneous and continuous Doppler radar velocity time series at the same altitude for at least three beams pointing in nonparallel directions. Then the frequency spectra and cross spectra can be computed from these beams. Analysis of the spectral slopes can determine the frequency power index, $p$. The Brunt Vaisala frequency, $w_{b}$, might also be estimated from these spectra or by other means such as from background wind and temperature profiles. The inertial frequency can be computed from the earth's rotational period and latitude of the radar site.

Using a 5-beam configuration where beam 1 points upward in the vertical direction, beams 2 and 4 obliquely to the east and west at an angle $q_{B}$, respectively, and beams 3 and 5 obliquely to the north and south at an angle $\mathrm{qB}_{\mathrm{B}}$, respectively. If we denote the velocity covariances measured by the ith beam as $\left\langle\mathrm{V}_{\mathrm{i}}{ }^{2}\right\rangle$, then the covariances for the wave associated velocity components can be computed from the $\left\langle\mathrm{V}_{\mathrm{i}}{ }^{2}\right\rangle$ :

$$
\begin{aligned}
& <\mathrm{W}^{2}>=\left\langle\mathrm{V}_{\mathrm{i}}^{2}>\right. \\
& <\mathrm{U}^{2}>=\left(<\mathrm{V}_{2}^{2}>+<\mathrm{V}_{4}^{2}>-2<\mathrm{W}^{2}>\cos ^{2} \theta_{\mathrm{B}}\right) /\left(2 \sin ^{2} \theta_{\mathrm{B}}\right) \\
& <\mathrm{V}^{2}>=\left(<\mathrm{V}_{3}^{2}>+<\mathrm{V}_{4}^{2}>-2<\mathrm{W}^{2}>\cos ^{2} \theta_{\mathrm{B}}\right) /\left(2 \sin ^{2} \theta_{\mathrm{B}}\right) \\
& <\mathrm{UW}>=\left(<\mathrm{V}_{2}^{2}>-<\mathrm{V}_{4}^{2}>\right) /\left(2 \sin ^{2} \theta_{\mathrm{B}}\right) \\
& <\mathrm{VW}>=\left(<\mathrm{V}_{3}^{2}>-<\mathrm{V}_{5}^{2}>\right) /\left(2 \sin ^{2} \theta_{\mathrm{B}}\right)
\end{aligned}
$$

If a sixth beam is added, say at $\phi_{6}=45^{\circ}$, then it is possible to obtain

$$
\langle U V\rangle=\frac{\left\langle V_{6}{ }^{2}\right\rangle-(\sqrt{2}+1)\left(\left\langle V_{2} 2\right\rangle+\left\langle V_{3}^{2}\right\rangle\right) / 4+\frac{\sqrt{2}-2}{c}\left(\left\langle V_{4}{ }^{2}\right\rangle+\left\langle V_{5}{ }^{2}\right\rangle\right) / 4}{\sin ^{2} \theta_{B}}
$$

With these six measured variances in eq. (11), the solution of eq. (9) allows for the quantitative estimation of four parameters for the model spectrum: $E_{0}, a,+\tan 2 \phi_{a}$, and $\left|S_{v h}\right|=\left|S_{v}\right|$ $\mathrm{S}_{\mathrm{h}}$. Under the set of assumptions made here, this leaves two degrees of freedom since there are six velocity variances in all. Due to this redundancy, it is possible to check the consistence of the wave spectrum hypothesis by computing some of the parameters in more than one way.

Let us first examine the case of $E_{0}$. Inspection of (9) shows that it is determined by $\left\langle W^{2}\right\rangle$, or $\left\langle\mathrm{U}^{2}\right.$. and $\left\langle\mathrm{V}_{2}\right\rangle$,

$$
\left.\mathrm{E}_{\mathrm{o}}=\frac{3-\mathrm{p}}{\mathrm{p}-1}\left(\frac{\omega_{\mathrm{p}}}{\omega_{\mathrm{i}}}\right) \mathrm{p}-1<\mathrm{W}^{2}\right\rangle \text {, or } \mathrm{E}_{\mathrm{o}}=\frac{\mathrm{p}+1}{2 \mathrm{p}}\left(\left\langle\mathrm{U}^{2}\right\rangle+\left\langle\mathrm{V}^{2}\right\rangle\right)
$$

The second way to obtain $\mathrm{E}_{\mathrm{o}}$ from velocity variances is from the horizontal components. If $\left\langle\mathrm{W}_{2}\right\rangle$ is obtained directly from a vertical radar measurement it may be contaminated with a horizontal velocity component depending on the possible sloped layer and the precise nature of the 
scattering mechanism. On the other hand, the value of $E_{0}$ obtained by decomposition of oblique velocity variance measurements can be compared to the one found by $\left\langle W^{2}\right\rangle$ to check the effect of the contamination. Greater consistency could be achieved for the parameter estimation scheme applied here if $\left\langle W^{2}\right\rangle$ were also determined by decomposition from an oblique beam configuration. The scattering mechanisms for multiple beams at a fixed oblique zenith angle are much likelier to be caused by the same process than the vertical beam. This is especially the case of $\theta_{\mathrm{B}}$ greater than about $2.5^{\circ}$ where turbulent scatter dominates. A way to obtain $\left\langle W^{2}\right\rangle$ from oblique measurements is to add the 7 th beam at a different beam angle $\theta_{\mathrm{B}}$ ' with $\phi_{\mathrm{B}}$, say, at $135^{\circ}$. Then

$$
<W^{2}>=\frac{A \sin ^{2} \theta_{B^{\prime}}-B \sin ^{2} \theta_{B}}{\sin \left(\theta_{B}{ }^{\prime}-\theta_{B}\right) \sin \left(\theta_{B}{ }^{\prime}+\theta_{B}\right)}
$$

where

$$
\begin{aligned}
& \left.A=\frac{1}{4}\left[\left\langle V_{2}{ }^{2}\right\rangle+\left\langle V_{3}^{2}\right\rangle+V_{4}{ }^{2}\right\rangle+\left\langle V_{5}{ }^{2}\right\rangle\right] \\
& B=\frac{1}{2}\left[\left\langle V_{6}^{2}\right\rangle+\left\langle V_{7}{ }^{2}\right\rangle\right]-\frac{\sqrt{2}}{4}\left[\left\langle V_{3}^{2}\right\rangle-\left\langle V_{5}{ }^{2}\right\rangle\right]\left(\frac{\sin 2 \theta_{B}{ }^{\prime}}{\sin 2 \theta_{B}}\right)
\end{aligned}
$$

Even if the observed velocity variances are obtained under identical scattering mechanisms, a discrepancybetween $E_{0}$ computed from (12) could still arise if the mesoscale atmospheric energy spectrum $E(k, \omega)$ is not entirely due to waves. The presence of two-dimensional atmospheric turbulence could contribute to a significant increase in the horizontal variance components without having an effect on the vertical variance which would still be due mainly to wave fluctuations. This provides a way to examine the contribution of 2-D turbulence to the overall observed velocity fluctuations.

The azimuth width factor, a, can be solved from (9). The most straightforward solution is:

$$
\frac{1-\mathrm{a}}{1+\mathrm{a}}=\frac{\left[\left(\langle\mathrm{U}\rangle-\left\langle\mathrm{V}^{2}\right\rangle\right)^{2}+(\langle\mathrm{UV}\rangle)^{2}\right]^{1 / 2}}{\left\langle\mathrm{U}^{2}\right\rangle\left\langle\mathrm{V}^{2}\right\rangle}
$$

Note that for the case of an azimuthally isotropic spectrum, such that $\mathrm{a}=1$ and so $\left\langle\mathrm{U}^{2}\right\rangle-\left\langle\mathrm{V}^{2}\right\rangle=$ $\langle U V\rangle=\langle U W\rangle=\langle V W\rangle=0$. Then the remaining parameters, $\phi_{\mathrm{a}}$ and $S_{\mathrm{vh}}$ cannot be determined by velocity variances alone. However, if $0 \leq \mathrm{a} \leq 1$, then we can continue this approach to solve these two parameters also.

The solution to $\phi_{\mathrm{a}}$ is quite straightforward.

$$
\tan 1 \phi_{\mathrm{a}}=\frac{2\langle\mathrm{UV}\rangle}{\left\langle\mathrm{U}^{2}\right\rangle-\left\langle\mathrm{V}^{2}\right\rangle} \text { or } \tan \phi_{\mathrm{a}}=\frac{\langle\mathrm{VW}\rangle}{\langle\mathrm{UW}\rangle}
$$

The two possible solutions to $\phi_{\mathrm{a}}$ cannot be distinguished by variances alone without other knowledge or an assumption about the sign of $S_{\mathrm{v}}$. A spectrum which consists of mainly downward phase propagation will, for example, have a negative $S_{v}$, and then (9) can be used to determine the quadrant of $\phi_{\mathrm{a}}$. The magnitude of the vertical-horizontal propagation factor $S_{\mathrm{vh}}$ can be found for $a \neq 1$ through the vertical fluxes of the zonal and meridional momenta. 


$$
\left|S_{v h}\right|=\left|S_{v}\right| S_{h}\left(\frac{1+a}{1-a}\right)^{1 / 2}\left(\frac{\omega_{b}}{\bar{\omega}}\right)\left[\left\langle U w^{2}\right\rangle+\left\langle v^{2}\right\rangle\right]^{1 / 2} / E_{0}
$$

This completes all the possible ways to estimate the parameters of the general model spectrum by using the nine variance components. Although these variances do include the momentum fluxes, they do not determine all the parameters in our model. Spatial information must also be available in order to determine $k_{z}{ }^{*}$ and $S_{v}\left(\right.$ or $S_{h}$ ). A vertical or nearly vertical observed wave number spectrum can be directly applied to estimate $\mathbf{k}_{\mathbf{z}}$ * such as was done by Scheffler and Liu [1985]. However, a two-dimensional data set is required to completely determine the propagation factors $S_{\mathrm{y}}$ or $S_{\mathrm{h}}$. The energy density content of each of the two branches of an observed $k_{z}-\omega$ velocity spectrum could, for instance, be used to estimate $S_{v}$. This parameter could also be determined from the same data set, but in the time domain by using the phase propagation correlation technique.

\section{CONCLUSIONS}

In this paper we derived the general expressions for various autospectra and cross spectra, as well as different types of variances for velocity fluctuations observed by multibeam MST radars. Assuming that these fluctuations are due to the presence of gravity waves, the radar-observed quantities are related to parameters of the general model gravity wave spectrum. These relations are used to design observational configurations such that the model parameters can be determined from the observed data.

The procedure can be summarized as follows. With one beam, pointing vertically, one can determine the spectral indices $\mathrm{p}$ and $\mathrm{t} ;$ also $\mathrm{E}_{\mathrm{o}}$, and possibly $\mathrm{S}_{\mathrm{y}}$ by $2-\mathrm{D}$ spectral analysis. With five beams, one vertical, two symmetrically off vertical in the E-W directions, and two $\mathrm{N}$-S directions, one can then determine the azimuthal asymmetry parameters, $a$, and the principal direction of the propagation ellipse, $\phi_{\mathrm{a}}$. With two additional beams, one with the same beam zenith angle but with different azimuth angle, one with different zenith angle, sufficient redundancy can be provided for checking the consistency of the assumption that the observed velocity fluctuations are due to gravity waves.

Finally, it should be mentioned that the major effort in this procedure is based on the variances of the observed quantities. This in many ways is more suitable for the purpose in determining model parameters than procedures that use spectral information alone.

\section{ACKNOWLEDGMENT}

The work described in this paper was supported by the National Science Foundation under grant ATM 8704947.

\section{REFERENCES}

B. B. Balsley, and D. A. Carter, The spectrum of atmospheric velocity fluctuations at $8 \mathrm{~km}$ and 86 km, Geophys. Res.. Lett, 9, 465-468, 1982.

C. E. Meek, I. M. Reid, and A. H. Manson, Observations of mesospheric wind velocities. II. Cross sections of power spectral density for $48-8 \mathrm{~h}, 8-1 \mathrm{~h}, 1 \mathrm{~h}-10 \mathrm{~min}$ over $60-110 \mathrm{~km}$ for 1981, Radio sci., 20, 1383-1402, 1985.

A. O. Scheffler, and C. H. Liu, On observation of gravity wave spectra in the atmosphere by using MST radar, Radio Sci., 20, 1309-1322, 1985.

A. O. Scheffler, and C. H. Liu, The effects of Doppler shift on gravity wave spectra observed by MST radar, J. Atmos. Terr. Phys., 48, 1225-1231, 1986.

S. A. Smith, D. C. Fritts, and T. E. VanZandt, Comparison of mesospheric wind spectra with a gravity wave model, Radio Sci., 20, 1331-1338, 1985.

S. J. Franke, C. H. Liu, I. J. Fu, R. Ruster, P. Czechowsky, and G. Schmidt, Multi-beam radar observations of winds in the mesosphere, submitted to .Geophys. Res., 1988.

T. E. VanZandt, A model for gravity wave spectra observed by Doppler sounding systems, Radio Sci., 20, 1323-1330, 1985. 